\title{
Comment on "Solute-specific scaling of inorganic nitrogen and phosphorus uptake in streams" by Hall et al. (2013)
}

\author{
R. González-Pinzón ${ }^{1}$, J. Mortensen ${ }^{1}$, and D. Van Horn ${ }^{2}$ \\ ${ }^{1}$ Department of Civil Engineering, University of New Mexico, Albuquerque, New Mexico 87131, USA \\ ${ }^{2}$ Department of Biology, University of New Mexico, Albuquerque, New Mexico 87131, USA \\ Correspondence to: R. González-Pinzón (gonzaric@unm.edu)
}

Received: 11 February 2015 - Published in Biogeosciences Discuss.: 23 April 2015

Revised: 3 September 2015 - Accepted: 3 September 2015 - Published: 17 September 2015

\begin{abstract}
Hall et al. (2013) presented a synthesis on 969 nutrient tracer experiments conducted primarily in headwater streams (generally $<$ fourth-order streams), with discharges $<200 \mathrm{~L} \mathrm{~s}^{-1}$ for $\sim 90 \%$ of the experiments, and used a scaling method to test the hypothesis that nutrient demand is constant with increasing stream size (i.e., along a river continuum). In this comment we present a reanalysis of a subset of the data used by Hall et al. (2013) and propose that their correlations between nutrient uptake lengths of ecologically important solutes and specific discharge are inadvertently spurious. Therefore, the conclusions derived from such correlations are debatable. We conclude the comment by highlighting some of the uncertainties associated with using modeling frameworks for scaling nutrient uptake in stream ecosystems.
\end{abstract}

\section{Estimating uptake lengths: transport model used by Hall et al. (2013)}

Hall et al. (2013) analyzed a data set of in-stream nutrient uptake experiments performed using plateau tracer injections. The basis of these experiments and estimation of nutrient uptake metrics come from the advection-dispersion equation (Eq. 1), with the addition of a first-order uptake rate coefficient (Stream Solute Workshop, 1990; Runkel, 2007):

$$
\frac{\mathrm{d} c}{\mathrm{~d} t}=-u \frac{\mathrm{d} C}{\mathrm{~d} x}+D \frac{\mathrm{d}^{2} C}{\mathrm{~d} x^{2}}-K_{\mathrm{c}} c,
$$

where $c\left(\mathrm{ML}^{-3}\right)$ is the concentration of the reactive solute at a cross section located downstream of the solute injection site; $u\left(\mathrm{LT}^{-1}\right)$ is the mean flow velocity; $D\left(\mathrm{LT}^{-2}\right)$ is the dispersion coefficient; $K_{\mathrm{c}}\left(\mathrm{T}^{-1}\right)$ is the first-order rate coefficient representing nutrient uptake; $x(\mathrm{~L})$ is longitudinal distance; and $t(\mathrm{~T})$ is time. Assuming that dispersion is negligible at plateau concentrations (i.e., when $\mathrm{d} c / \mathrm{d} t=0$ ), Eq. (1) can be solved for downstream solute concentration:

$c=c_{o} \exp \left(-\left(K_{\mathrm{c}} / u\right) x\right)$,

where $c_{o}\left(\mathrm{M} \mathrm{L}^{-3}\right)$ represents the initial (or upstream) concentration. The form of this solution motivated the introduction of the uptake length metric, $S_{w}=u / K_{\mathrm{c}}$, which is a representation of the average distance traveled by a nutrient molecule in inorganic phase prior to uptake (Ensign and Doyle, 2006). Due to experimental simplicity, Eq. (2) has guided data collection efforts on nutrient cycling where an experimentalist estimates $S_{w}$ by measuring the plateau concentrations upstream $\left(c_{\text {up }}\right)$ and downstream $\left(c_{\mathrm{dn}}\right)$ of a study reach of length $L:$

$S_{w}=u / K_{\mathrm{c}}=L / \ln \left(C_{\mathrm{up}} / C_{\mathrm{dn}}\right)$.

Note that Eqs. (1-3) support estimates of $S_{w}$, given that stream conditions satisfy model assumptions, i.e., that stream reaches have constant discharge and that dispersion and transient storage do not play important roles (Runkel, 2007). The uptake length derived from Eqs. (1) to (3) is equivalent to $S_{w}^{\mathrm{I}}$ in Runkel (2007), who derived four different uptake lengths $\left(S_{w}^{\mathrm{I}}, S_{w}^{\mathrm{II}}, S_{w}^{\mathrm{III}}, S_{w}^{\mathrm{IV}}\right)$ from solute transport models with increased complexity (i.e., with added transient storage, lateral inflows and dispersion). Following Runkel (2007), uptake lengths can be generally represented by a velocity term and an uptake term.

It is important to keep in mind that $S_{w}$ is an abstract variable represented by model parameters that cannot be simultaneously measured. Since $u$ and $K_{\mathrm{c}}$ are likely to be highly 
Table 1. The relationship $S_{w}$ vs. $Q / w$ for natural-channel geometries.

\begin{tabular}{lll}
\hline $\begin{array}{l}\text { Quantity or } \\
\text { relationship }\end{array}$ & $\begin{array}{l}\text { Rectangular } \\
\text { channel }\end{array}$ & $\begin{array}{l}\text { Non-rectangular } \\
\text { channel }\end{array}$ \\
\hline$A$ & $w \times h$ & $f(w, h)$ \\
$Q$ & $u \times A$ & $u \times A$ \\
$S_{w}$ & $u / K_{\mathrm{c}}$ & $u / K_{\mathrm{c}}$ \\
$Q / w$ & $u \times h$ & $u \times(f(w, h) / w)$ \\
$S_{w}$ vs. $Q / w$ & $u / K_{\mathrm{c}}$ vs. $u \times h$ & $u / K_{\mathrm{c}}$ vs. $u \times(f(w, h) / w)$ \\
\hline
\end{tabular}

variable along a stream reach, measurements of longitudinal decline in tracer concentrations $\left(c_{\mathrm{up}}, c_{\mathrm{dn}}\right)$ and stream length $(L)$ offer a more tractable approach to estimating $S_{w}$ through the use of Eq. (3). While the use of Eq. (3) circumvents errors associated with estimating $u$ and $K_{\mathrm{c}}$ on the reach scale, the estimation of $S_{w}$ using $c_{\mathrm{up}}, c_{\mathrm{dn}}$, and $L$ must be numerically equivalent to $u / K_{\mathrm{c}}$ on the reach scale. As is the case with any abstract variable derived from a mathematical model, using $S_{w}$ to infer stream processes entails acknowledging the quantitative role of the model parameters $u$ and $K_{\mathrm{c}}$ from where $S_{w}$ was derived.

\section{Critique of the scaling approach used by Hall et al. (2013)}

The analysis presented by Hall et al. (2013) was based on plateau experiments conducted in multiple stream ecosystems, where $S_{w}$ was estimated for each experiment using Eq. (3). Hall et al. correlated nutrient uptake length, $S_{w}(\mathrm{~L})$, with specific discharge, $Q / w\left(\mathrm{~L}^{2} \mathrm{~T}^{-1}\right)$, to test the hypothesis that nutrient uptake demand is constant across stream orders.

$S_{w} \propto(Q / w)^{a}$

$v_{f}=\frac{Q / w}{S_{w}}$

where $Q\left(\mathrm{~L}^{3} \mathrm{~T}^{-1}\right)$ is stream discharge, $w(\mathrm{~L})$ is stream width, $a$ is a scaling exponent and $v_{f}\left(\mathrm{~L} \mathrm{~T}^{-1}\right)$ is the nutrient uptake demand (or nutrient uptake velocity, as it has been traditionally called).

In their hypothesis testing, the existence of a constant nutrient uptake demand (constant $v_{f}$ ) was implied by a scaling exponent $a=1$ (isometric scaling), whereas a scaling exponent $a \neq 1$ (allometric scaling) would imply the reverse. Note that in this context, the existence of a constant nutrient uptake demand would be useful to scale and predict nutrient uptake in stream ecosystems.

In Table 1 we present the different forms that $S_{w}$ vs. $Q / w$ from Hall et al. (2013) would take if such a relationship was estimated for two general types of natural-channel geometries.

Note that each side of $S_{w}$ vs. $Q / w$ shares the common (hidden) variable $u$. Therefore, an increase in $u$ (e.g., with stream order or increasing discharge) would increase both sides of the proportion, likely forcing a strong correlation between the variables. This would happen regardless of whether $u$ is measured in the field or not because $S_{w}$ is an abstract quantity derived from $u$ and $K_{\mathrm{c}}$ (cf. Eqs. 2-3), and, by definition, $Q=u \times A$. The fact that Hall et al. (2013) used only estimates of $S_{w}$, and field measurements of $Q$ and $w$ to seek a mechanistic relationship from $S_{w}$ vs. $Q / w$ (cf. Eq. 4) does not change the induced correlation created by having the factor $u$ playing a key quantitative role on both sides of the relationship. Since the form of $S_{w}$ is dependent on the transport model presented in Eqs. (1-2), the only way to negate the role of $u$ in $S_{w}$ (note that it cannot be negated in $Q / w$ ) is to select a completely different transport model and perform a completely different set of field experiments. Also, under the ideal scenario in which we could actually measure $S_{w}$ in streams (i.e., if $S_{w}$ was not an abstract variable), the regression $S_{w}$ vs. $Q / w$ would mainly support the development of conceptual models for $S_{w}$, which already exist.

We propose that if a meaningful, significant correlation exists between $S_{w}$ and $Q / w$, there should be a significant correlation between the underlying parameters (i.e., $1 / K_{\mathrm{c}}$ vs. $h$ in rectangular channels or $1 / K_{\mathrm{c}}$ vs. $f(w, h) / w$ in other types of natural channels). However, if there is not a corresponding correlation in both of these cases, then the correlation between $S_{w}$ and $Q / w$ would be falsely influenced by the presence of $u$ in both products. Benson (1965) and Kenney (1982) demonstrated that spurious correlations can result from the use of ratios or products that share a common factor and are more likely when working with complex variables and dimensional analysis. The relationship from Hall et al. (2013) that we deem spurious is analogous to that of Model II presented by Benson (1965) for the spurious correlation of products sharing a common factor (i.e., $X_{1} \times X_{2}$ vs. $X_{3} \times X_{2}$; where $X_{1}=1 / K_{\mathrm{c}}, X_{2}=u, X_{3}=h$ or $X_{3}=f(w, h) / w$; cf. Table 2 in Benson, 1965). As shown by Benson (1965), the correlation of complex variables (i.e., $S_{w}$ and $\left.Q / w\right)$ is dependent on the coefficients of correlation and variation of the three original component variables. Due to the presence of a common factor in the scaling relationship proposed by Hall et al. (2013), we hypothesize that it is a spurious correlation ( $u$ influences both $S_{w}$ and $Q / w$ ) that may be mechanistically irrelevant for scaling in-stream nutrient uptake.

We tested our hypothesis using the data set published by Tank et al. (2008), another meta-analysis of nutrient addition experiments, which was included in the Hall et al. (2013) meta-analysis. This data set was chosen because it reports values for $S_{w}, Q, w$, and $h$ for nutrient experiments with $\mathrm{NH}_{4}$ and $\mathrm{NO}_{3}$ (SRP - soluble reactive phosphorus - not included), even though these values were not reported for all the studies $\left(n=143\right.$ for $\mathrm{NH}_{4} ; n=210$ for $\left.\mathrm{NO}_{3}\right)$. Note that since we do not know the particular geometry for each channel where the tracer experiments were conducted (i.e., we do not know $f(w, h)$ ), we assumed a rectangular chan- 


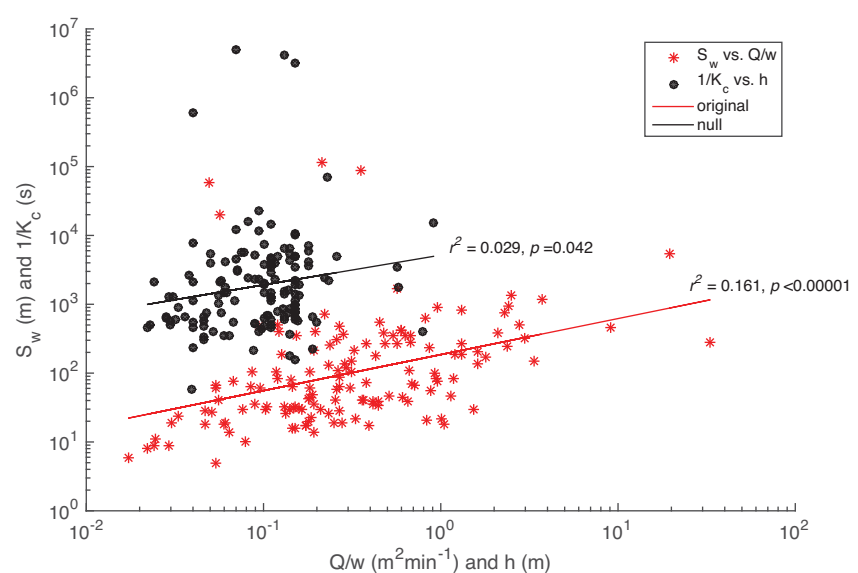

Figure 1. $\mathrm{NH}_{4}$ scaling relationship with and without shared velocity term. The original relationship is represented by $S_{w}$ vs. $Q / w$ and the null condition by $1 / K_{\mathrm{c}}$ vs. $h$.

nel geometry (i.e., $A=f(w, h)=w h)$, which is the same assumption as that made by Hall et al. (2013), while defining their equations for uptake length and uptake velocity (cf. Eqs. 1-2 in Hall et al., 2013). The data set published by Hall et al. (2013) does not include values of $h$; hence, we were not able to use it for our analysis. While the assumption of having rectangular channels might be seen as an overgeneralization, it is the only one that allows us to see trends given the scarce information available on the channel geometries of the headwater streams where the experiments were conducted. Furthermore, the transport model implicitly used by Hall et al. (2013) assumes uniform flow (i.e., $\mathrm{d} h / \mathrm{d} x=0$; $\mathrm{d} w / \mathrm{d} x=0)$, which supports our assumption of a prismatic channel for testing our spurious-correlation hypothesis.

We proposed a null condition in which we removed the common variable $u$ from the scaling relationship and compared the correlation with that of the original scaling relationship (i.e., we compared $1 / K_{\mathrm{c}}$ vs. $h$ and $S_{w}$ vs. $Q / w$ ). We calculated mean stream velocity as $u=Q /(w \times h)$. This allowed us to produce values for the relationship $1 / K_{\mathrm{c}}$ vs. $h$, by dividing $S_{w}$ and $Q / w$ by $u$ (cf. Table 1 ). By doing so, we were able to evaluate the scaling relationship with and without the common term $u$ to compare the coefficient of determination, $r^{2}$, for both relationships. Results of this analysis are shown for $\mathrm{NH}_{4}$ and $\mathrm{NO}_{3}$ in Figs. 1 and 2.

Our results show that $1 / K_{\mathrm{c}}$ vs. $h$ are weakly correlated $\left(r_{\left(\mathrm{NH}_{4}\right)}^{2}=0.029, \quad p_{\left(\mathrm{NH}_{4}\right)}=0.042 ; r_{\left(\mathrm{NO}_{3}\right)}^{2}=0.036\right.$, $\left.p_{\left(\mathrm{NO}_{3}\right)}=0.0057\right)$. However, the correlation $S_{w}$ vs. $Q / w$ is higher $\left(r_{\left(\mathrm{NH}_{4}\right)}^{2}=0.161, p_{\left(\mathrm{NH}_{4}\right)}<0.00001 ; r_{\left(\mathrm{NO}_{3}\right)}^{2}=0.151\right.$, $\left.p_{\left(\mathrm{NO}_{3}\right)}<0.00001\right)$, i.e., $r^{2}$ is improved by 452 and $317 \%$ for $\mathrm{NH}_{4}$ and $\mathrm{NO}_{3}$, respectively. These findings suggest that the correlation $S_{w}$ vs. $Q / w$ is spurious because it is driven by the shared velocity $(u)$ term rather than by an inherent correlation between the inverse of the nutrient uptake rate constant $\left(1 / K_{\mathrm{c}}\right)$ and stream depth $(h)$. The correlations shown

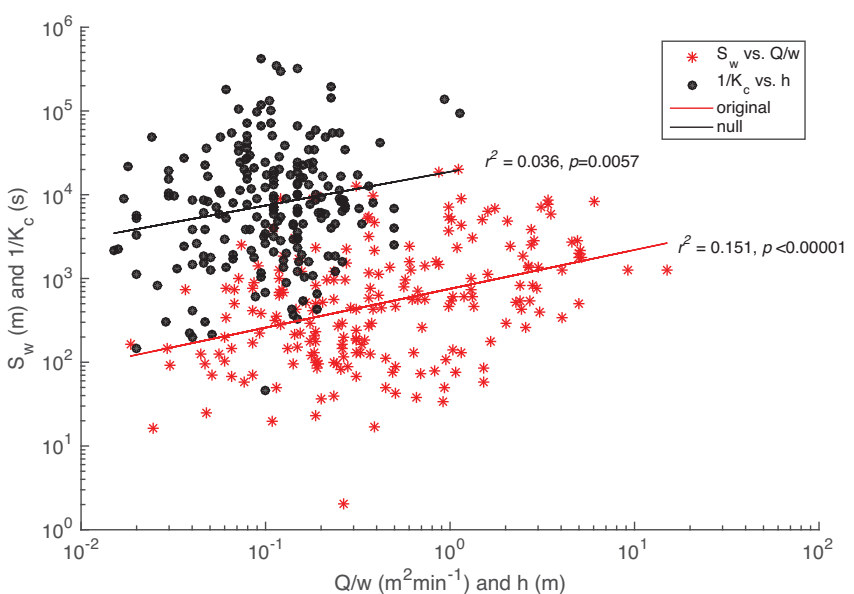

Figure 2. $\mathrm{NO}_{3}$ scaling relationship with and without shared velocity term. The original relationship is represented by $S_{w}$ vs. $Q / w$ and the null condition by $1 / K_{\mathrm{c}}$ vs. $h$.

in Figs. 1 and 2 are comparable to those reported by Hall et al. (2013). However, we note that the $r^{2}$ values do not match because of different data sets (we were limited by the number of studies reporting all parameters $S_{w}, Q, w, h$ ) and our aggregation of reference and altered streams. Regardless, our analysis suggests that the inclusion of the parameter $u$ falsely improves the correlation of the investigated relationships.

The mechanism producing spurious correlation in the data set by Hall et al. (2013) can be viewed more clearly using three arbitrary and uncorrelated variables to represent the relationship between $X_{1} \times X_{2}$ and $X_{3} \times X_{2}$. We gathered mean daily values for specific conductance $\left(X_{1}, \mu \mathrm{S} \mathrm{cm}^{-1}\right)$ in the Potomac River (DC) (USGS, 2008a), turbidity $\left(X_{2}\right.$, FNU) in the Little Arkansas River (KS) (USGS, 2008b), and temperature $\left(X_{3},{ }^{\circ} \mathrm{C}\right)$ in the Rio Grande (NM) (USGS, 2008c) for the year 2008. First, we isolated the common factor $X_{2}$ and plotted $X_{1}$ versus $X_{3}$, as shown in Fig. $3\left(r^{2}=0.020\right.$, $p=0.012$ ). As expected, there was no statistically significant correlation between these water quality parameters. However, when we incorporated the turbidity $\left(X_{2}\right)$ from a remote location by plotting $X_{1} \times X_{2}$ vs. $X_{3} \times X_{2}(n=313)$, we found a positive correlation (Fig. 4) with a drastic improvement in $r^{2}\left(r^{2}=0.846, p<0.00001\right)$. Despite the evident correlation in this relationship, the result is mechanistically irrelevant. Analogous to this case example where the correlation is driven by $X_{2}$ (turbidity), the correlation $S_{w}$ vs. $Q / w$ seems to be driven by $u$ (recall $S_{w}=u K_{\mathrm{c}}$ and $Q / w=u \times h$ or $Q / w=u \times(f(w, h) / w))$. Thus, our findings suggest that the results produced by Hall et al. (2013) regarding the isometric scaling $(a=1)$ of $\mathrm{NH}_{4}$, and allometric scaling $(a>1)$ of $\mathrm{NO}_{3}$ and SRP, resulted from an unintentional spurious correlation of $S_{w}$ vs. $Q / w$.

In addition to scaling nutrient uptake length with specific discharge, Hall et al. (2013) also provide a method for scaling nutrient uptake with stream length using several parameters, 


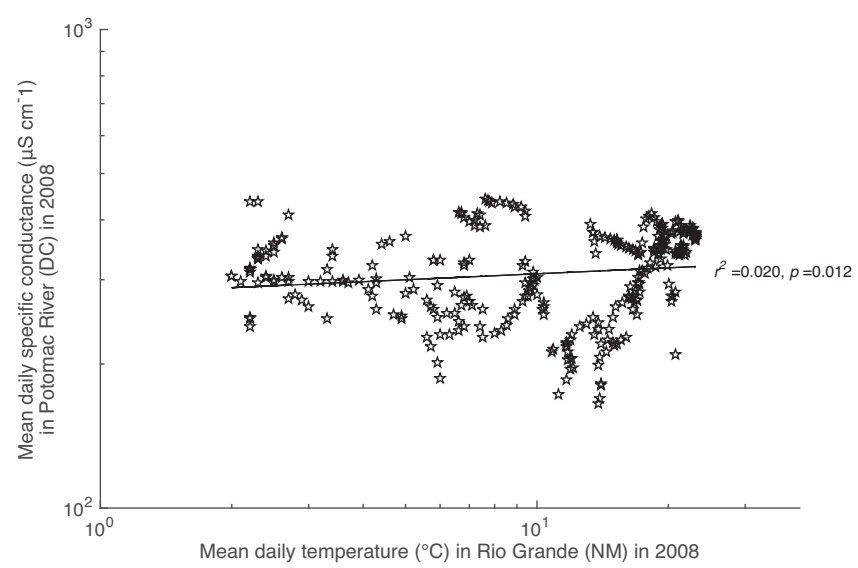

Figure 3. Synthetic data correlation, type $X_{1}$ vs. $X_{3}$, without common parameter, $X_{2}$. There is a weak correlation between these water quality parameters.

including the scaling exponent $a$ obtained from the analysis of the scaling relationship shown in Eq. (2). Our findings have implications for these results as well. While Hall et al. (2013) commented that their results for scaling uptake with stream length were most influenced by $b$ (hydraulic geometry exponent), their analysis still relies on the spurious correlation $S_{w}$ vs. $Q / w$ not only for parameter $a$ but also for the subsequent derivations (cf. Eqs. 3-10 in Hall et al., 2013). Therefore, we also find those results debatable.

\section{Concluding remarks}

The majority of nutrient addition experiments have been performed in headwater streams because they are more experimentally tractable (Tank et al., 2008). Consequently, the dearth of empirical evidence of nutrient processing in large rivers limits our understanding of the role of these rivers in nutrient processing on the catchment scale. While empirical and theoretical advances are being made toward performing nutrient addition experiments in large rivers (Tank et al., 2008; Covino et al., 2010), the need to understand and quantify nutrient export from these systems has driven the development and use of scaling relationships. This motivated the work by Hall et al. (2013), and their results after correlating $S_{w}$ vs. $Q / w$ for a large data set of field nutrient experiments suggest that uptake demand $\left(v_{f}\right)$ for $\mathrm{NH}_{4}$ is relatively constant across stream orders, whereas that for soluble reactive phosphorous (SRP) and $\mathrm{NO}_{3}$ declines with increasing specific discharge. Here, we demonstrated that these conclusions are subject to debate due to unintentional spurious correlations present in their scaling relationships.

We also suggest that $S_{w}$ should be used with extreme caution to scale nutrient uptake because, even though its magnitude can be directly estimated from relatively simple field measurements, its mechanistic interpretation strongly

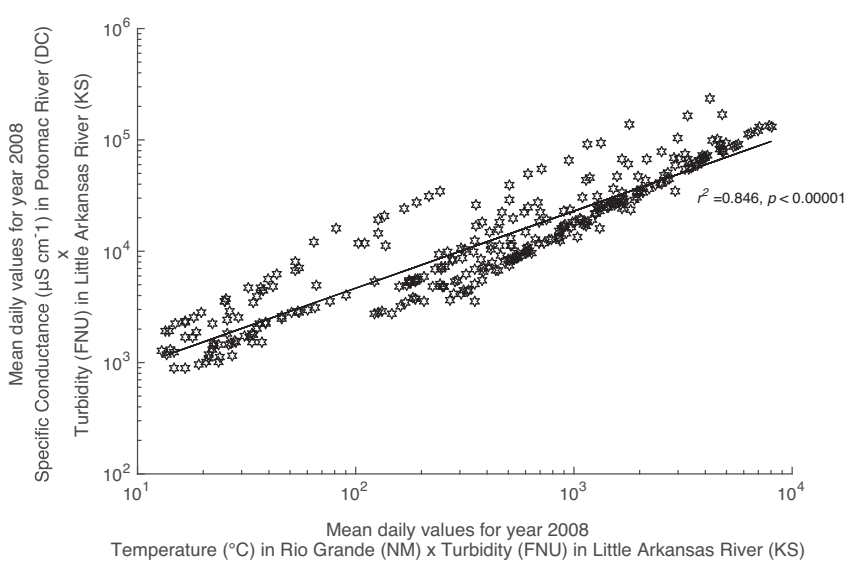

Figure 4. Synthetic data correlation, type $X_{1} \times X_{2}$ vs. $X_{3} \times X_{2}$, with common parameter $X_{2}$. This spurious correlation results simply because $X_{2}$ is common to both quantities.

depends on the type of model assumed to describe the realworld system (cf. Table 1 in Runkel, 2007). This is because the same estimate of the magnitude of $S_{w}$ may be arbitrarily used to co-estimate or constrain the magnitude of parameters describing different (arbitrary) sets of processes (see Cases IIV in Runkel, 2007). Finally, when a model describing a given set of processes is chosen to interpret how nutrient uptake scales along a river continuum, the main assumption is that such processes operate analogously along the continuum. For example, if the model of advection-decay chosen by Hall et al. (2013) to interpret $S_{w}$ across stream orders were correct, our analysis presented in Figs. 1 and 2 would suggest that headwater streams tend to have higher nutrient uptake rate coefficients, which might be mechanistically supported by their higher ratio of benthic area to cross-sectional area. However, this (biased) analysis would not provide insight into how mass-transfer processes between the main-channel and transient storage zones may control nutrient uptake and retention along the river continuum. Paradoxically, increasing the complexity of the transport models used to derive $S_{w}$ (e.g., Cases II-IV in Runkel, 2007) does not necessarily improve the mechanistic understanding gained on how nutrient uptake scales along the river continuum because such models are poorly constrained, i.e., the number of parameters introduces more degrees of freedom than the data collected (from field and remote measurements) can constrain.

Acknowledgements. We want to thank Gabriel Huerta and Tim Covino for providing feedback on earlier versions of this manuscript. This work was funded by NSF grant HRD-1345169.

Edited by: K. Fennel 


\section{References}

Benson, M. A.: Spurious correlation in hydraulics and hydrology, J. Hydraul. Div., 91, 35-42, 1965.

Covino, T. P., McGlynn, B. L., and McNamara, R. A.: Tracer Additions for Spiraling Curve Characterization(TASCC): Quantifying stream nutrient uptake kinetics from ambient to saturation, Limnol. Oceanogr., 8, 484-498, 2010.

Ensign, S. H. and Doyle, M. W.: Nutrient spiraling in streams and river networks, J. Geophys. Res., 111, G04009 doi:10.1029/2005JG000114, 2006.

Hall Jr., R. O., Baker, M. A., Rosi-Marshall, E. J., Tank, J. L., and Newbold, J. D.: Solute-specific scaling of inorganic nitrogen and phosphorus uptake in streams, Biogeosciences, 10, 7323-7331, doi:10.5194/bg-10-7323-2013, 2013.

Kenney, B. C.: Beware of spurious self-correlations, Water Resour. Res., 18, 1041-1048, 1982.

Runkel, R. L.: Toward a transport-based analysis of nutrient spiraling and uptake in streams, Limnol. Oceanogr., 5, 50-62, 2007.
Stream Solute Workshop: Concepts and Methods for Assessing Solute Dynamics in Stream Ecosystems, J. N. Am. Benthol. Soc., 9, 95, doi:10.2307/1467445, 1990.

Tank, J. L., Rosi-Marshall, E. J., Baker, M. A., and Hall, R. O.: Are rivers just big streams? A pulse method to quantify nitrogen demand in a large river, Ecology, 89, 2935-2945, 2008.

US Geological Survey: National Water Information System data available on the World Wide Web (USGS Water Data for the Nation), available at: http://waterdata.usgs.gov/dc/nwis/dv? referred_module $=$ sw\&site_no=_01646500 (last access: 7 January 2015), 2008a.

US Geological Survey: National Water Information System data available on the World Wide Web (USGS Water Data for the Nation), available at: http://waterdata.usgs.gov/ks/nwis/dv? referred_module $=\_$sw\&site_no $=\_07144100$ (last access: 7 January 2015), 2008b.

US Geological Survey: National Water Information System data available on the World Wide Web (USGS Water Data for the Nation), available at: http://waterdata.usgs.gov/nm/nwis/uv?site_ no=_08317400 (last access: 7 January 2015), 2008c. 